\title{
Supporting Collaborative Work in Socio-Physical Environments: A Normative Approach
}

\author{
Catherine Garbay and Fabien Badeig and Jean Caelen
}

\begin{abstract}
We propose a normative approach to collaborative support system design in distributed tangible environments. Based on activity theory, our goal is to mediate rather than drive human activity and to integrate components from the physical, numerical and social environments. We propose an original architecture coupling a physical space (tools supporting human activity), a processing space (agent performing activity, be it human or artificial), an informational space (traces reflecting activity), and a normative space (filters regulating activity). We further consider collaboration as a conversational process grounded in the objects of the working space. To this end, tangible objects of various kinds are designed to support multi-threaded activity. Heterogeneous trace properties may then be fused to situate activity and ground the filtering process. Interface agents are designed to provide appropriate visual feedback and support mutual awareness. Beyond the mere sharing of individual or group activity, we approach awareness as involving mutual knowledge of the activity constraints. We show through simple examples from the RISK game the potential richness of the proposed approach.
\end{abstract}

Key words: Collaboration architectures ; Tangible Distributed Interfaces ; Activity Theory ; Normative Multi-Agent Systems

\section{Introduction}

We propose in this chapter a normative approach to design collaborative support system in distributed tangible environments, in the framework of the TangiSense infrastructure [Lepreux et al., 2011]. In accordance with activity theory, the guiding principles of our design are (1) to mediate rather than drive human activity, (2) to

Badeig, Garbay, Caelen

LIG, Université de Grenoble, UFR IM2AG - BP 53 - F-38041 Grenoble Cedex 9, e-mail: \{first_name.last_name\}@imag.fr 
articulate within a single framework tools from the physical environment and rules from the social realm. Collaboration in our design does not amount to sharing the most visible part of activity, but further involve the mutual understanding of laws and rules that forge its background in more or less explicit ways. In addition, we propose that sharing be addressed in the framework of privacy constraints that may be placed at the individual or group level. Another guiding principle is to recognize the dynamic nature of both environments: the components of activity such as tools, goals and rules are constantly changed, constructed and transformed in relation to the outcome of the activity system.

To this end, we propose an original architecture coupling four spaces: a physical space (tools supporting human activity, be it tangible objects or virtual displays), a processing space (agent performing activity, be it human or artificial), an informational space (traces reflecting activity), and a normative space (filters regulating activity). In this framework, the trace embeds the mutual relationships between tools, agents, and constraints from their social environment; agents are responsible for the activity dynamics while filters implementing the norms are responsible for its regulation. Traces are meant to register activity as well as its compliance to the systems of norms at hand, to reflect the constraints tying the collaborative effort. They may in turn be communicated or transformed, thus mediating future activities. To support rich conversational exchange from distant places, within the constraint of tangible environments, we propose to embody communication within tangible objects and virtual displays representing various threads of activity. Collaboration may then be seen as a conversational process grounded in the objects of the working space. The fusion of trace properties then allows situating activity and grounding the filtering process. Interface agents are designed to provide appropriate visual feedback and support mutual awareness. The degree of awareness is further modulated by privacy constraints regulating the extent to which the activity is to be tracked and made visible. We show through simple examples from the RISK game the potential richness of the proposed approach.

\section{Application Framework}

The TangiSense table [Lepreux et al., 2011] may be seen as a magnetic retina which is able to detect and locate tangible objects equipped with RFID tags (Figure 1). This retina is made of 25blocks containing 64antennas and readers of one square inch each. Each block of antennas further contains two microprocessors, one dedicated to the management of the RFID readers and another one to the handling of Ethernet communications. The density of antennas allows a spatial and temporal resolution compatible with the real-time detection of moving objects by users. The blocks are driven by the Infrastructure Layer running on the PC host. The role of this layer is to deal with the infrastructure communication, to filter potentially unstable tags IDs and positions, or as well to aggregate information in case a tag is crossing between two blocks. When RFID tags are pasted underneath tangible objects, it is possible 
(i) to detect their presence on the surface of the table, (ii) to proceed to their identification, since RFID tags are unique and (iii) to store relevant information directly in the object tag's memory (for example their last position, or their last owner). Each RFID antenna is further equipped with 4 multicolor light emitting diodes (leds). The primary role of these diodes is to provide feedback, that is to react to tangible objects positioning and moves, assessing for the user their effective detection by the table. Their secondary role is to provide additional information to the user, from the mere assessment of action correctness, to the display of color pattern enriching the feedback to the user by indicating places where events occur.

The Risk game is a strategic board game for two to six players. The standard version is played on a board depicting a political map of the Earth, divided into forty-two territories, which are grouped into six continents. Upon start, each player is allocated an army (cannons, soldiers, cavalrymen) and a set of territories that his army occupies. He is further assigned a mission card specifying his target, be it to conquer some territories or to defeat a given army. An attack takes place according to a well-defined process: the attacking player first of all designates two territories, the first one, from his own territories, supporting the attacking armies, and the second one, from the board, being attacked. The attacking player defines the number of attacking armies in moving army pieces. The attacking and attacked players then throw dices to determine who is loosing or winning this round. The attacking player may conduct as many attacks as wanted: the assault continues until he decides to retire, or until one of the two is eliminated. The attacking player may finally operate some army moves, before it is the turn of the other player. A player is eliminated in case all his territories have been lost. The game is finished as soon as one player has reached its mission goal. Figure 1 display example views of the RISK game, as played on the TangiSense table. The potential interest of this application, as regards our proposed modeling, is to offer a static environment of limited complexity, with players operating at the same organizational level, under strict coordination rules. In this context, the goal of the proposed design is to offer a coordination framework ensuring smooth interplay, considering that the players have no communication means except the table and its tangible and virtual equipments. We will illustrate our modeling approach in section 6 , in the framework of three main functionalities: initial setting up of the players, management of player privacy and management of interplayer coordination.

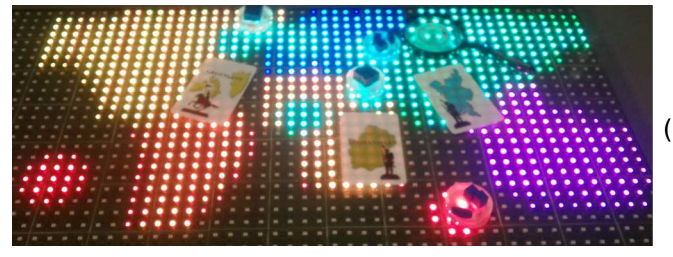

(a)

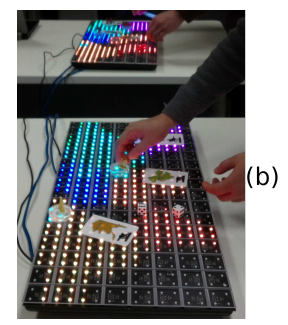

Fig. 1 (a) The TangiSense table as equipped for the RISK game with tangible objects and virtual led displaying the ground map as well as tangible objects moves; (b) distributed view: objects handling is transmitted in real time, whatever the distance between the two tables. 


\section{State of the art}

To design collaborative support systems, a major issue is to preserve the spontaneity and fluidity of human activity while ensuring the consistency and proper coordination of action [Pape and Graham, 2010]. We consider these issues in the framework of activity theory and normative multi-agent system design, with the goal to integrate in a unified view the physical, numerical and social realms of human activity. Activity theory articulates within a single dynamics the individual and social components of human activity (the subject, the object and the tool, on the one hand, the group, its organization and rules, on the other hand). According to this theory, the object is seen and manipulated not as such but within the limitations set by the instrument [Engestrom, 1991]. The tool restricts the interaction to be from the perspective of that particular tool or instrument only [Kuutti, 1995]. It mediates the structure and objectives of activity; it is in turn transformed and built along this activity and therefore keeps track of user experience [Bourguin et al., 2001]. As quoted by [Nardi, 1996], "the structuring of activity is not something that precedes it but can only grow directly out of the immediacy of the situation". The involvement in action create circumstances that could not anticipated in advance, and the object and motive reveal themselves only in the process of doing. As a consequence, the limitations set by the instrument may not be modeled a priori, rather they reveal in the course of action. A tool can be physical, mental, or semiotic, it can be a heuristic that one follows to transform an object, or it can be a speech act that transforms a situation [Cole, 1996]. Communication may therefore be thought of as grounded in the objects of the working space, which may be designed as coordination objects and raise action. [Kraut et al., 2003] for example analyzes the role of visual information as a conversational resource in performing collaborative tasks, to maintain mutual awareness, ground conversation and facilitate mutual understanding. According to [Shaer and Hornecker, 2010], tangible objects might be used as full resources to support epistemic action, be it oriented toward the physical or numerical world (reference to situate action, sensorial or perceptual resource to sustain involvement into action, ...). Coordination is considered as driven implicitly by [Sire and Chatty, 1998], thanks to an increased consideration of human coordination capacities, and to the search for a balance between the handling of affordant objects and the application of implicit communication norms. Activity theory further recognizes the dynamic nature of context, i.e. the fact that the activity tools, goals and rules are constantly changed, constructed, and transformed in relation to the outcome of this activity [Cole, 1996, Greenberg, 2001]. Context is defined as the coupling of two main components, one internal (mental context, private objectives) and the other external (physical and social environment in which activity develops) [Dumazeau and Karsenty, 2008]. According to [Lewandowski, 2006], an integrated computer-supported collaboration environment should provide, configure and handle for each actor the set of tools he needs, accounting for his rights and duties for this activity.

Mutual awareness both of the state of task objects and of one another's activities [Kraut et al., 2003] is a core issue to sustain collaboration; it is defined as shar- 
ing mutually manifest information or events [Salembier and Zouinar, 2004], and implying belief in mutual attention [Dumazeau and Karsenty, 2008]. Physical copresence provides multiple resources for awareness and conversational grounding (e.g., sights, smells, touch) which have to be complemented in the case of distant communication. Traces of activity have been proposed by several researchers as a way to represent, share and visualize human experience in its interaction with numerical platforms. Interaction traces have further been explored to enhance synchronous collaboration, and sharing traces at a group level has been advocated to support group awareness [Clauzel et al., 2011]. The notion of social translucence has been proposed by [Erickson and Kellogg, 2003] to represent the degree to which distant actors share the constraints potentially limiting their activity, in terms of visibility, awareness and accountability. A new tool called social proxy is proposed in this respect: these graphical representations display socially salient aspects of some situation (distant talks, bidding, queuing ...) with a variable degree of visibility.

Normative agents have been proposed by the COIN (Coordination, Organization, Institutions and Norms in Agent Systems) community to face the conceptual antagonism between the autonomy of a system and its control. Coordination in this approach is seen as a social paradigm: agent behavior is not only guided by their mere individual objectives but also regulated by norms specifying which actions are considered as legal or not by the group. The norms may operate at several levels in the system, they specify in a declarative way actions considered as legal or accepted. They may be adopted or not by the agents, which may result in penalties, and adapted to cope with the evolution of context [Boella et al., 2007, Boissier et al., 2011]. The system dynamics therefore depends not only on the agent dynamics but also on the dynamic of the norms. The approach has been recently extended to specify the interaction modes of agents belonging to the same organization [Boissier et al., 2011]. The goal of such specification is to provide tools allowing to reason on these interaction modes and to check their appropriateness. Another major issue is to maintain consistency, especially in contexts where human actors do not know each other, are communicating from distant places, and may display opposite or conflicting goals. An application to the control of multi-player computer games has been studied in [Gâteau et al., 2006]. The purpose is to constrain players and their avatars to adopt a team-like behavior and to respect rules, while allowing some autonomy to keep the game appealing. One further requirement concerns the evolution of the game, since rules change according to rounds of the game. The proposed design articulates two layers using a normative organizational model: the multi-agent interactive game in which avatars evolve as autonomous agents, and an institutional multi-agent middle-ware called SYNAI (SYstem of Normative Agents for Institution) dedicated to the management of the organization and to the arbitration. The role of this arbitration system consists in rewarding or punishing agents when they respect or not their agreements. Finally mention is made in [Okuyama et al., 2008] of a distributed and situated approach to normative design. The proposed normative infrastructure is composed of normative objects and normative places, and further allows the spatial contextualization of norms. 


\section{Proposed View}

Human activity is multi-threaded: collaboration may be seen as a conversational act, where the signs of dialog are multiple (moving, designing, acknowledging, referring, turn taking, giving speech). These signs of dialog are missing in the course of distant collaboration. To benefit from the tangibility offered by the infrastructure at hand, we propose to design dedicated tangible objects, called tangigets, to support distant communication and use them to produce signs and traces of conversational activity. We will for example distinguish between a designation tangiget (a tool to delineate zones on the table surface or points out an object, thus indicating its relevance to distant actors), an annotation tangiget (a tool to express acceptance or rebuttal of a given utterance), and a coordination tangiget (a tool to indicate the starting or ending of activities). A privacy tangiget has been added to account for modifications in the privacy rules.

Collaborative activity does not reduce to exchanging views, or sharing the production of results. More deeply, it amounts to sharing common goals and intentions, and to understand the physical or social constraints surrounding activity. Privacy rules may in addition rule out the degree of sharing. These constraints are often kept implicit and thus remain invisible; they vary from one individual to the other (preferences, own objectives), from one organization to the other (rules for turn taking or for conducting operations), and may as well depend on the current goal or physical environment. Some of these rules may hold for the whole collaborative session or evolve according to the processing stage or as well depending on individual decisions.

To cope with these various issues, we ground our design on an extended notion of trace, where traces reflect both human activity and its relationship to the norms under consideration, through a set of property values and types. The norms are represented explicitly via filters deposited in the system environment, which may evolve along the various stages of collaboration or more opportunistically in case of some tangible object moves (privacy tangiget for example). Filters are triggered in a situated way, via the fusion of trace properties expressing a pattern and context of activity. They further enrich these trace properties by reflecting their compliance to the norms.

They finally participate to the system regulation by launching agents, whose role is to keep track of tangible objects moves and process the trace accordingly, to generate feedback to the users and to maintain the system of norms at hand by removing or depositing filters. Interface agents are designed to provide appropriate visual feedback. Feedback is operated in a dual way, to reflect activity (virtual display reflecting tangible object moves on distant tables), and to reflect norm compliance (virtual display underneath a tangible object reflecting the legitimacy of its move to the actor responsible for this move). Such feedback is regulated by privacy rules.

In this framework, norms do not act as a prerequisite to action or as a way to prevent action. Rather, they allow grounding the physical infrastructure of the collaborative environment into the realm of a social organization. These norms are not static, but may evolve, to account for dedicated contexts or demands. They further 
reveal in the course of action in the form of new trace properties, a set of signs revealing potentially complex relationships and facets, which will afterwards be interpreted, possibly in different contexts by different actors.

\section{Proposed Architecture}

We finally model collaboration as a situated process coupling four spaces: the physical space (human activity tools, be it tangible objects or virtual displays), the informational space (activity traces), the processing space (activity performers, be it human or artificial agents) and the normative space (activity regulation rules). Four kinds of elements are therefore distinguished in the proposed architecture (Figure 2): (1) tools, i.e. tangible objects and their virtual representatives, that appear in the physical environment; (2) traces, i.e. numerical objects, which keep track of human and artificial agent activity and register the privacy of this information as well as its compliance to the system of norms at hand; (3) actors, i.e. human and artificial agents, whose role is (i) to handle tools, thus generating and updating traces of activity, and (ii) to handle the normative space, i.e. to deposit filters in the system; (4) filters, condition-action rules, which regulate the system activity by (i) modifying the privacy of trace properties, (ii) checking the compliance of activity with respect to the system of norms at hand and (iii) launching agents to react to trace modifications.

In this framework, human and artificial agents are responsible for the activity dynamics while filters implementing the norms are responsible for the regulation of this dynamics. Traces evolve jointly under the regulatory action of filters and the processing action of human and artificial agents, which are operated in an asynchronous and concurrent way. To the dynamics of traces corresponds a dynamics of norms, deposited along the collaboration process. Filters are deposited by agents and may be set as active or inactive. Active filters are repeatedly checked for application to the information space (traces), under control of a dedicated engine (not shown in the figure). They launch agents as soon as some pattern of interest is detected over the information traces.

When activated, the system operates according to the following information flow (also shown in Figure 3): (1) Early detection of a tangible or tangiget object move by event filters operating at the infrastructure level: creation or update of the corresponding local trace; (2) Triggering of the consistency and privacy filters: update of the corresponding local traces; (3) Triggering of the tangible, tangiget and interface filters: computation of some local trace property, feedback to local and distant human actors.

We distinguish between four types of agents: (1) Tangible agents: their role is to perform the computation required by the application and enrich the trace properties (decision resulting from dice roll results for example); (2) Tangiget agents: depending on the tangiget that has been handled, their role is to launch the requested coordination policy, that it is to modify the policy at hand by depositing the corresponding 


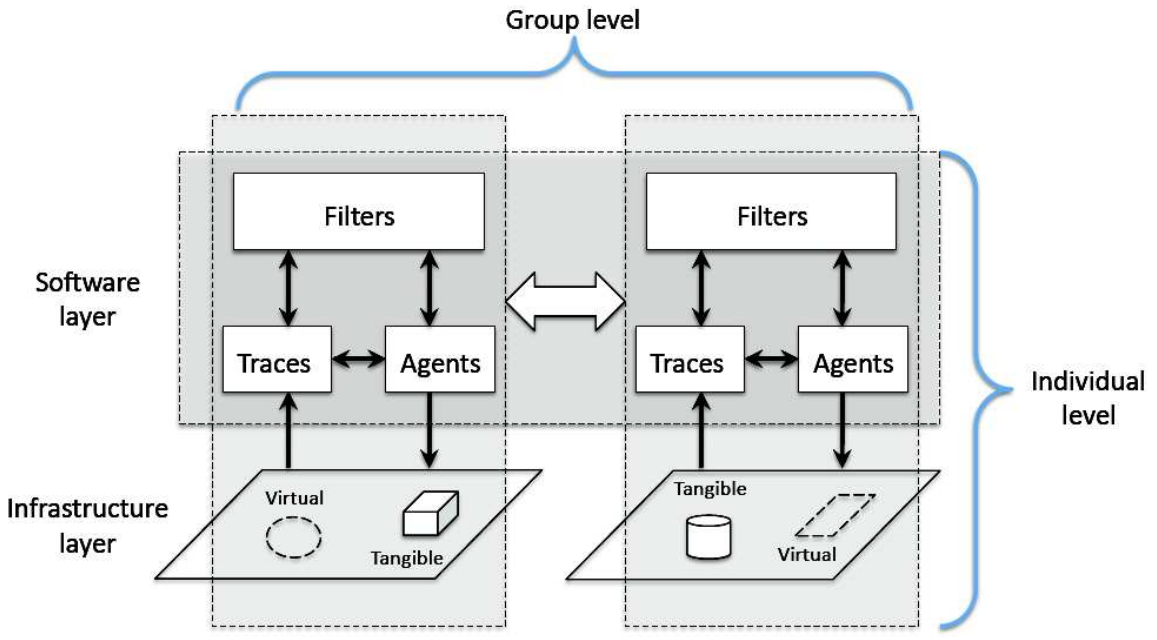

Fig. 2 Collaboration as the joint management of traces (representing activity), norms (regulating activity) and agents (performing activity).

set of filters; (3) Interface agents: their role is to provide visual feedback, by means of led display, informing either about a distant tangible object move, about the result of consistency analysis (consistency of tangible object move, result of consistency filter) or about a request (request for a tangible object move, result of a coordination filter); (4) Global agents further operate on some global trace properties, under the regulation of dedicated global filters, to maintain the directory of actors and for the sake of global processing consistency (they are not displayed in Figure 3, for the sake of simplicity).

The advantage of distant collaboration is to benefit from a free and private space of action that may not be accessed from the outside. Another key element is the possibility to continue active working in phases where one has to wait for results from distant tables, by drafting in a concrete way various potential intermediate solutions and keeping them private. Some privacy rules may therefore hold for the whole collaborative session or evolve according to the collaboration stage or to individual decisions. We therefore propose (i) that private/public spaces be delimitated from start by means of dedicated privacy filters (in the RISK game for example, the player mission card must be kept private) and (ii) to dynamically open and (re)open these spaces as soon as it is decided or necessary for the course of action (in the RISK game for example, a player may want to simulate some attacks and keep them private before entering the full play). This kind of closing/opening action may for example be ruled by means of a dedicated tangiget.

Traces in our present design are attached to tangible and tangiget objects, to describe their properties and current state. They are further attached to the virtual representatives of these objects. Any trace is considered as a set of (property, value) pairs. We propose that properties be typed, to register their privacy and compliance to the norms. As a consequence, a trace is expressed as follows: 


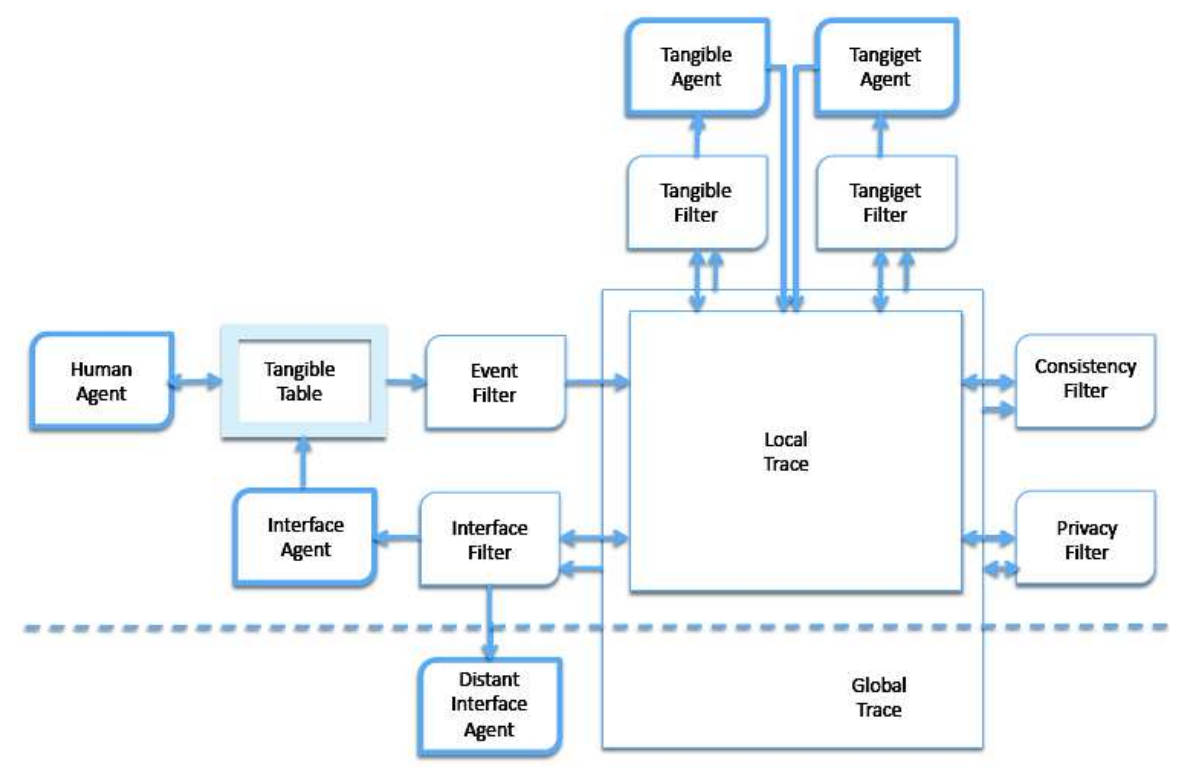

Fig. 3 Functional view showing the various types of agents, filters and traces.

$$
\begin{aligned}
& \text { Trace }=\{(p, v)\} \text { where } p=\langle\text { name }: \text { consistency }: \text { privacy }\rangle \\
& \text { with consistency } \in\{\text { valid, invalid }\} \text { and privacy } \in\{\text { private, } \text { public }\}
\end{aligned}
$$

Valid (resp. invalid) expresses the fact that the given property is compliant (resp. not compliant) with respect to the norms at hand. Private (resp. public) means that the property is not accessible (resp. accessible) to distant agents. Newly created traces are considered as private, with a non-assigned (null) consistency. As soon as some trace property is public, it becomes accessible to distant filters.

Any trace is defined as possessing the following minimal properties (identifying name, type of tangible object, tangible table where originated, spatial position on this table, time of move). The type of a trace may be either tangible, tangiget or abstract (to refer to global properties of the whole system of tables).

We distinguish between five kinds of filters (Figure 3): (1) Event filters: filters that regulate the creation of traces from stream of events generated by objects move on the table surface; these filters are part of the infrastructure layer and not considered into more details in the following; (2) Interface filters: filters that launch interface agents as soon as some trace is created or modified (under the constraint that privacy rules hold); (3) Tangible (resp. tangiget) filters: filters that launch tangible (resp. tangiget) agents; (4) Consistency filters: filters that check the compliance of human activity with respect to some norms, and thus enrich the trace via the field consistency of trace properties; (5) Privacy filters: filters that modify the trace local visibility and accessibility to distant actors, and thus enrich the trace via the field privacy of trace properties. 


\section{An Application to the RISK Game}

The goal of this section is to illustrate the potential of the proposed design and provide some concrete hints regarding representation and processing issues. We ground this analysis on an application to the RISK game that has been presented in section 2. We focus on three activity/regulation stages (player identification, privacy, attack coordination protocol) exemplifying different aspects of our modeling.

Player Identification - The player identification process starts as soon as a player handles his player identification card and puts in place his coordination tangiget. Handling this tangible object results in the creation of a trace, which is described in Table 1.

\begin{tabular}{|l|l|l|l|}
\hline Property.name & Property.consistency & Property.privacy & Value \\
\hline Type & Null & Private & (player card, tangible) \\
OnTable & Null & Private & Table1 \\
Name & Null & Private & Dupont \\
Id & Valid & Private & 2 \\
Position & Null & Private & $(0.3,0.5)$ \\
\hline
\end{tabular}

Table 1 Description of the player trace.

Such creation triggers a validation filter $F_{\text {consistency }}$ whose role is to check whether this player is legitimate or not and modify the player trace accordingly. $F_{\text {consistency }}$ is described in Table 2.

\begin{tabular}{|c|c|c|c|c|c|c|}
\hline$F_{\text {consistency }}$ & Property name & Property field & Parameter & Operator & Value & \\
\hline Conditions & Type & & ?Trace 1 & $=$ & (game, abstract) & (1) \\
\hline & PlayerNumber & & ?Trace 1 & $<$ & & (2) \\
\hline & Type & & ?Trace 2 & $=$ & (player card, tangible) & (3) \\
\hline & Type & Consistency & ?Trace 2 & $=$ & Null & (4) \\
\hline & Id & & ?Trace 2 & $=$ & & (5) \\
\hline & Type & & ?Trace 3 & $=$ & (coordination, tangiget) & (6) \\
\hline & Status & & ?Trace 3 & $=$ & Initialization & (7) \\
\hline & Handled & & ?Trace 3 & $=$ & ?id & (8) \\
\hline Actions & \multicolumn{5}{|c|}{$\forall i, p_{i}$.consistency $(?$ trace 2$) \leftarrow$ Valid } & (9) \\
\hline
\end{tabular}

Table 2 Description of the $F_{\text {consistency }}$ filter elements.

As may be seen, the condition part of this filter involves the composition of heterogeneous trace elements: global trace of the game, to check the number of players already entered in the game (must be under 6), status of the player's coordination tangiget (must be "Initialization"), presence of a new player identification card.

An agent attached to the follow-up of this new player is then created, thanks to a new filter, $F_{n e w}$ player , described in Table 3 . The role of this filter is to launch the player agent and the coordination agent, whose role is to ensure any further processing for this new player.

Privacy - As regards privacy, the default policy is that any player (or player action) be known and visible to other players. However, this policy holds under the 


\begin{tabular}{|c|c|c|c|c|c|c|}
\hline$F_{\text {new player }}$ & Property name & Property field & Parameter & Operator & Value & \\
\hline \multirow[t]{9}{*}{ Conditions } & Type & & ?Trace 1 & $=$ & (player card, tangible) & $(1)$ \\
\hline & Position & Consistency & ?Trace 1 & $=$ & Valid & $(2)$ \\
\hline & Position & & ?Trace 1 & $=$ & ?pos & (3) \\
\hline & OnTable & Consistency & ?Trace 1 & $=$ & Valid & (4) \\
\hline & OnTable & & ?Trace 1 & $=$ & $? t$ & (5) \\
\hline & & & ?Trace 1 & $=$ & & $(6)$ \\
\hline & Type & & ?Trace 2 & $=$ & (coordination, tangiget) & (7) \\
\hline & Status & & ?Trace 2 & $=$ & Initialization & (8) \\
\hline & Handled & & ?Trace 2 & $=$ & ?id & (9) \\
\hline Actions & \multicolumn{5}{|c|}{$\begin{array}{l}\text { launch }\left(\text { agent }_{\text {coordination }}, ? t, \text { succeed }\right. \\
\text { launch }\left(\text { agent }_{\text {plaver }}, ? t, ? \text { Trace } 1\right)\end{array}$} & $\begin{array}{l}(10) \\
(11)\end{array}$ \\
\hline
\end{tabular}

Table 3 Description of the $F_{\text {new }}$ player filter elements.

condition that the corresponding information or action are valid, according to the norms at hand. This basic policy may further be changed by handling a dedicated tangiget, to declare some action as private (not discussed further in this section).

We have shown in Table 1, the example trace of a newly created player, with some properties kept private. We give in Table 4, the description of a filter, called $F_{\text {privacy, }}$, whose role is to transform these trace properties, under the condition that the current stage of the player's coordination tangiget is "Initialization".

\begin{tabular}{|l|l|l|l|l|l|l|}
\hline$F_{\text {privacy }}$ & Property name & Property field & Parameter & Operator & Value & \\
\hline Conditions & Type & & ?Trace 1 & $=$ & (player card, tangible) & $(1)$ \\
& Type & Consistency & ?Trace 1 & $=$ & Valid & $(2)$ \\
& Type & Privacy & ?Trace 1 & $=$ & Private & $(3)$ \\
& Id & ?Trace 1 & $=$ & ?id & $(4)$ \\
& Type & ?Trace 2 & $=$ & (coordination, tangiget) & $(5)$ \\
& Status & ?Trace 2 & $=$ & Initialization & $(6)$ \\
& Handled & & ?Trace 2 & $=$ & ?id & $(7)$ \\
\hline Actions & $\forall i, p_{i}$ privacy $(?$ Trace 1$) \leftarrow$ Public & $(8)$ \\
\hline
\end{tabular}

Table 4 Description of the $F_{\text {privacy }}$ filter elements.

As soon as the player trace is valid and public, a new filter may be triggered, whose role is to launch the Interface agent in order to provide feedback of this new event to distant tables. This filter, called $F_{\text {interface }}$, relies on a complex condition part, checking that the player trace properties are simultaneously valid and public, that the state of the coordination trace is "Initialization", and finally that distant player traces are valid and public.

Coordination - The purpose of this section is to illustrate inter-player coordination, based on a simple example from the RISK game: launching of an attack by a given player. According to our framework, inter-player coordination is ensured (i) by the players themselves handling dedicated tangigets, (ii) by the normative system expressing policies of action and (iii) by the visual feedback provided to each player. Two different tangigets are considered in the following: a coordination tangiget (a dice with sides representing various stages of the game) and a designation tangiget (to point out the attacking and attacked territories). 


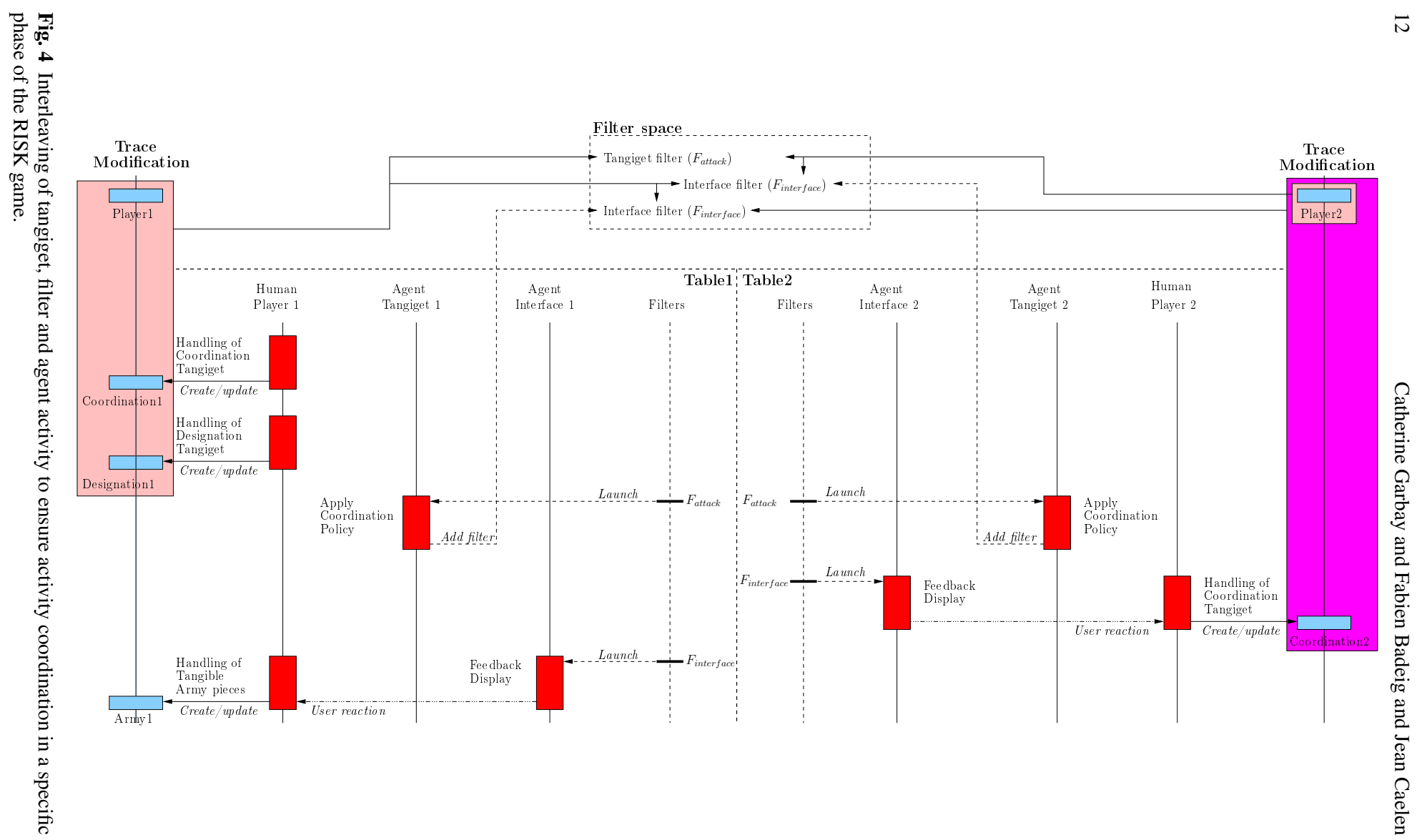


As exemplified in Figure 4, such coordination finally involves interleaved tangigets, filters and agents handling. Filter description may easily be derived from the descriptions in table 2, 3 and 4 . The whole process may be decomposed as follows:

- Tangiget handling: handling the coordination and designation tangigets modify the corresponding traces, which triggers the tangiget filter $F_{\text {attack }}$; this filter which launches tangiget agent 1 on the attacking player local environment together with tangiget agent 2 on the attacked player distant environment $\left(F_{\text {attack }}\right.$, acting at a global level in the game, is actually provided the rights for creating a relationship between both players and launching these agents);

- Policy management: it is then the role of both tangiget agents to deposit the policy they plan to follow, in the form respectively of the Interface filter $F_{\text {interface }}$;

- Communication management: the role of $F_{\text {interface }}$ is to launch interface agent 2 , so that information about the attack under preparation be provided to the distant player (lightning of leds underneath the distant player identification card, coordination tangiget and attacked territory); $F_{\text {interface }}$ condition part involves information about both distant players identification, coordination and designation traces;

- Tangiget handling: upon receipt of this feedback, player 2 reacts by handling his own coordination tangiget, thus acknowledging receipt of this action;

- Communication management: the role of $F_{\text {interface }}$ is now to launch interface agent 1, to transmit player 2's acknowledgement of receipt (lightning of leds underneath player 1 coordination and designation tangigets); $F_{\text {interface }}$ condition part involves information about both distant players identification, coordination and designation traces);

- Player 1 may now proceed forward by handling his fighting army.

\section{Overview of the correlations between agents and filters}

To show the system dynamics, we finally summarize in Table 5 the various types of filters and agents involved in this short scenario, together with their roles, triggering events, results and dependencies.

\section{Conclusion}

We have presented a design to support collaboration under tangible environments. Our core contribution is to provide a seamless integration between elements from the physical, numerical and social world, in a design grounded into normative multiagent system theory. Human collaboration in our design is mediated via tangible objects and registered via multi-dimensional numerical traces. These traces are made to evolve under the application of normative rules, to keep track not only of a given activity, but furthermore of the normative context surrounding this activity. Visual 


\begin{tabular}{|c|c|c|c|c|c|}
\hline $\begin{array}{l}\text { Interaction } \\
\text { scene }\end{array}$ & $\begin{array}{l}\text { Player } \\
\text { idenfication }\end{array}$ & Attack & Consistency & Privacy & Interface \\
\hline Role & $\begin{array}{l}\text { New player join- } \\
\text { ing the current } \\
\text { game }\end{array}$ & $\begin{array}{l}\text { Regulate inter- } \\
\text { player coordi- } \\
\text { nation during } \\
\text { attack state }\end{array}$ & $\begin{array}{ll}\text { Verify human } \\
\text { activity con- } \\
\text { sistency }\end{array}$ & $\begin{array}{l}\text { Update the } \\
\text { accessbility of } \\
\text { traces }\end{array}$ & $\begin{array}{lr}\text { Operate risual } \\
\text { feedback } & \text { on } \\
\text { distant table } & \end{array}$ \\
\hline Filter & $F_{\text {new }}$ plaver & $F_{\text {attack }}$ & $F_{\text {consistency }}$ & $F_{\text {privacy }}$ & $F_{\text {interface }}$ \\
\hline \begin{tabular}{|l|} 
Trigger \\
Components
\end{tabular} & $\begin{array}{l}\text { Tangible Object: } \\
\text { Player } \\
\text { Tangiget: } \\
\text { Coordination }\end{array}$ & $\begin{array}{l}\text { Tangible Object: } \\
\text { Player } \\
\text { Tangiget: } \\
\text { Coordination } \\
\text { Designation }\end{array}$ & $\begin{array}{l}\text { Property: } \\
\text { Consistency }\end{array}$ & $\begin{array}{l}\text { Property: } \\
\text { Privacy }\end{array}$ & $\begin{array}{l}\text { Tangible Object } \\
\text { Tangiget } \\
\text { Property: } \\
\text { Consistency } \\
\text { Privacy }\end{array}$ \\
\hline Action-Trace & $\emptyset$ & Modify traces & Modify traces & Modify traces & $\emptyset$ \\
\hline Action-Agent & $\begin{array}{l}\text { Launch: } \\
\text { Agent }_{\text {Player }} \\
\text { Agent }_{\text {Coordination }}\end{array}$ & $\begin{array}{l}\text { Launch: } \\
\text { Agent }_{\text {Attack }} \\
\text { Agent }_{\text {Coordination }} \\
\text { Agent }_{\text {Designation }}\end{array}$ & $\emptyset$ & $\emptyset$ & $\begin{array}{l}\text { Launch: } \\
\text { Agent Interface }\end{array}$ \\
\hline Dependencies & Consistency & \begin{tabular}{|l|} 
Player \\
identification
\end{tabular} & $\emptyset$ & $\emptyset$ & $\begin{array}{l}\text { Consistency } \\
\text { Privacy }\end{array}$ \\
\hline $\begin{array}{l}\text { Consequences } \\
\text { at the agent } \\
\text { level }\end{array}$ & $\begin{array}{l}\text { Subscribe to } \\
\text { local and global } \\
\text { policies in terms } \\
\text { of consistency, } \\
\text { privacy and } \\
\text { interface }\end{array}$ & $\begin{array}{l}\text { Add appropriate } \\
\text { privacy, consis- } \\
\text { tency, interface } \\
\text { filters for this } \\
\text { specific phase }\end{array}$ & $\overline{\mid \eta}$ & 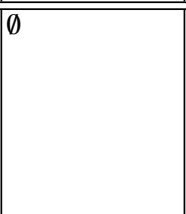 & $\overline{\mid \eta \emptyset}$ \\
\hline
\end{tabular}

Table 5 Correlations between agents and filters in the short RISK game scenarios.

feedback is further provided, to reflect activity, in a way that is situated with respect to the norms at hand. Context awareness is therefore approached as involving both activity and norm awareness.

In accordance with the principles of activity theory, norms do not act as a prerequisite, or as a way to apply a priori constraints on action. Rather, they are meant to situate action, by evaluating properties that may then be considered by other agents, in proper contexts. Human collaboration in this context is mediated rather than assisted by complex computerized systems; it is regulated rather than constrained. Collaboration is finally defined as a process by which individual and collective norms are appropriated and co-evolve. Further specification and implementation, in front of a real application, is of course mandatory to more thoroughly investigate the potential, difficulties and limitations of this approach.

Acknowledgements Work supported by Agence Nationale de la Recherche (ANR) under grant IMAGIT - project ANR 2010 CORD 01701. 


\section{References}

[Boella et al., 2007] Boella, G., van der Torre, L. V. N., and Verhagen, H. (2007). Introduction to normative multiagent systems. In Normative Multi-agent Systems.

[Boissier et al., 2011] Boissier, O., Balbo, F., and Badeig, F. (2011). Controlling multi-party interaction within normative multi-agent organisations. In Coordination, Organization, Institutions and Norms in Agent Systems VI (LNAI 6541). Springer-Verlag.

[Bourguin et al., 2001] Bourguin, G., Derycke, A., and Tarby, J. (2001). Beyond the interface: coevolution inside interactive systems a proposal founded on activity theory. In IHM-HCI 2001 conference, People and computer XV - Interactions without Frontiers, pages 297-310. Springer Verlag.

[Clauzel et al., 2011] Clauzel, D., Sehaba, K., and Prié, Y. (2011). Enhancing synchronous collaboration by using interactive visualisation of modelled traces. In Simulation Modelling Practice and Theory, volume 19, pages 84-97.

[Cole, 1996] Cole, M. (1996). Cultural Psychology : A once and future discipline. Cambridge, MA.: Harvard University Press.

[Dumazeau and Karsenty, 2008] Dumazeau, C. and Karsenty, L. (2008). Communications distantes en situation de travail. In "Le travail humain", $P U F$, pages 225-252.

[Engestrom, 1991] Engestrom, Y. (1991). Developmental work research: reconstructing expertise through expansive learning. In Human jobs and computer interfaces, pages 265-290.

[Erickson and Kellogg, 2003] Erickson, T. and Kellogg, W. (2003). Social translucence: Using minimalist visualizations of social activity to support collective interaction. In Hk, K., Benyon, D., and Munroe, A., editors, Designing Information Spaces: The Social Navigation Approach, pages 17-41. Springer-Verlag.

[Gâteau et al., 2006] Gâteau, B., Boissier, O., Khadraoui, D., and Dubois, E. (2006). Controlling an interactive game with a multi-agent based normative organizational model. In Coordination, Organization, Institutions and Norms in agent systems workshop (COIN) at the 17th European Conference on Artificial Intelligence (ECAI), Italy.

[Greenberg, 2001] Greenberg, S. (2001). Context as a dynamic construct. Human-Computer Interaction, 16:257-268.

[Kraut et al., 2003] Kraut, R. E., Fussell, R., and Siegel, J. (2003). Visual information as a conversational resource in collaborative physical tasks. In $H C I$, pages 13-49.

[Kuutti, 1995] Kuutti, K. (1995). Activity theory as a potential framework for human-computer interaction research. In Context and Consciousness: Activity Theory and Human Computer Interaction, pages 17-44. Cambridge: MIT Press.

[Lepreux et al., 2011] Lepreux, S., Kubicki, S., Kolski, C., and Caelen, J. (2011). Distributed interactive surfaces using tangible and virtual objects. In Workshop DUI'2011 Distributed User Interfaces, at CHI'2011, pages 65-68, Canada.

[Lewandowski, 2006] Lewandowski, A. (2006). Vers de meilleurs supports aux activités coopératives en accord avec la co-évolution : application au développement logiciel coopératif. $\mathrm{PhD}$ thesis, Université du Littoral Côte d'Opale.

[Nardi, 1996] Nardi, B. (1996). Activity theory and human-computer interaction. In Context and consciousness: activity theory and human-computer interaction, pages 69-103. Cambridge and London, MIT Press.

[Okuyama et al., 2008] Okuyama, F., Bordini, R., and da Rocha Costa, A. (2008). A distributed normative infrastructure for situated multi-agent organisations. In Sixth International Workshop on Declarative Agent Languages and Technologies (DALT) to held as part of AAMAS-2008.

[Pape and Graham, 2010] Pape, J. and Graham, T. (2010). Coordination policies for tabletop gaming. In Graphics Interface, pages 24-25.

[Salembier and Zouinar, 2004] Salembier, P. and Zouinar, M. (2004). Intelligibilité mutuelle et contexte partagé. In Activités, page 6485.

[Shaer and Hornecker, 2010] Shaer, O. and Hornecker, E. (2010). Tangible user interfaces: Past, present and future directions. In $H C I$, pages 1-138.

[Sire and Chatty, 1998] Sire, S. and Chatty, S. (1998). Vers des interfaces à collaboration directe pour le travail de groupe. In Actes du Colloque GRESICO, page 259275. 